\title{
A pilot study of extremely low-frequency magnetic fields in advanced non-small cell lung cancer: Effects on survival and palliation of general symptoms
}

\author{
CHENGTAO SUN, HUIMING YU, XINGWEN WANG and JUNQING HAN \\ Department of Tumor Research and Therapy Center, Provincial Hospital Affiliated to Shandong University, \\ Shandong University, Shandong 250021, P.R. China
}

Received March 20, 2012; Accepted August 10, 2012

DOI: $10.3892 / \mathrm{ol} .2012 .867$

\begin{abstract}
The inhibitory effects of magnetic fields (MFs) on tumor cell proliferation in vitro and in vivo have been reported in previous studies. However, the effects of MFs in the treatment of cancer have not been described in clinical trials. We investigated the effects of $420 \mathrm{r} / \mathrm{min}$, 0.4-T extremely low-frequency MFs (ELF-MFs) on the survival and palliation of general symptoms in 13 advanced non-small cell lung cancer (NSCLC) patients. Toxicity and side-effects were assessed according to WHO criteria. The treatment area included the primary tumor site, metastatic sites and metastatic lymph nodes. Additionally, the patients were treated $2 \mathrm{~h}$ per day, 5 days per week for 6-10 weeks. The changes in general symptoms were analyzed during ELF-MF treatment and 2 weeks after the completion of therapy. Results of physical examination, routine analysis of blood, ECG and liver function, biochemical and kidney function tests were evaluated before and following treatment. All 13 patients were followed up by outpatient service or telephone interview. Our results demonstrated that decreased pleural effusion, remission of shortness of breath, relief of cancer pain, increased appetite, improved physical strength, regular bowel movement and better sleep quality was detected in $2(15.4 \%), 5(38.5 \%), 5(38.5 \%), 6(46.2 \%), 9(69.2 \%), 1$ $(7.7 \%)$ and $2(15.4 \%)$ patients, respectively. However, the palliation of symptoms in $2(15.4 \%)$ patients was observed during therapy and disappeared at treatment termination. No severe toxicity or side-effects were detected in our trial. The median survival was 6.0 months (95\% CI, 1.0-11.0). The 1and 2-year survival rates were 31.7 and $15.9 \%$, respectively.
\end{abstract}

Correspondence to: Professor Junqing Han, Department of Tumor Research and Therapy Center, Provincial Hospital Affiliated to Shandong University, Shandong University, 324 Jingwu Weiqi Road, Jinan, Shandong 250021, P.R. China

E-mail: hanjq1960@126.com

Key words: extremely low-frequency magnetic fields, non-small cell lung cancer, toxicity, survival analysis, general symptoms
This study is the first to describe survival and palliation of general symptoms in advanced NSCLC patients treated with ELF-MFs. As an effective, well-tolerated and safe treatment choice, ELF-MFs may prolong survival and improve general symptoms of advanced NSCLC patients. However, this treatment strategy requires further research.

\section{Introduction}

There has been growing interest in the effects of magnetic fields (MFs) on cancer research and therapy in recent years. Huang et al (1) investigated the effects of $20-\mathrm{mT}, 50-\mathrm{Hz}$ sinusoidal MFs on cell proliferation, ion concentration and osmolarity in two human cancer cell lines (HL-60 and SK-Hep-1). MFs inhibition of cell growth was observed and the authors concluded that these inhibitions in vitro may relate to changes in cell ion concentration and osmolarity. A synergistic effect was found when immunocompetent mice bearing murine Lewis lung carcinomas (LLCs) or B16 melanotic melanomas treated with cisplatin were exposed to extremely low-frequency (ELF)-MFs (2). Novikov et al (3) suggested that MFs have a marked antitumor activity. Mice with transplanted Ehrlich ascites carcinoma (EAC) were exposed to weak MFs and the tumor tissue was almost completely absent after exposure. Zhang et al (4) reported that ELF pulsed-gradient MFs induce apoptosis of cancer cells and arrest neoangiogenesis, inhibiting murine malignant tumor growth.

The mechanism of the anticancer activity of MFs is uncertain. Ronchetto et al (5) hypothesized that the free radical recombination processes were influenced by MFs, which activated p53 gene-dependent survival mechanisms. Elson (6) reported the production of numerous breaks in DNA and the overwhelming of DNA repair processes, leading to the apoptotic process. DNA damage was also considered as one of the possible mechanisms. Ruiz-Gómez and MartínezMorillo (7) reviewed 29 studies and concluded that MFs act as co-inductors of DNA damage.

Certain researchers have evaluated the toxicity of MFs. Tofani $\mathrm{S}$ et al (2) reported that no clinical signs or toxicity were observed in any mice exposed to MFs compared with mice administered cyclophosphamide or cisplatin. Sprague- 
Dawley rats were exposed to $20 \mathrm{kHz}$ triangular MFs in the study of Lee et al (8) and the authors concluded that MFs did not increase toxicity in rats. In clinical research, Ronchetto et al (5) assessed acute toxicity in patients with advanced neoplasia who accepted static MFs treatment and concluded that MFs may be safely administrated according to the exposure schedules.

In vivo and in vitro, the inhibition of MFs on tumor cell proliferation has been reported in numerous studies (9-12). However, thus far, clinical trials to evaluate the improvement of survival and general symptoms in advanced cancer patients treated with ELF-MFs have not been performed. Therefore, 13 advanced non-small cell lung cancer (NSCLC) patients were enrolled in this study. The aim of this study was to investigate the efficacy and safety of ELF-MFs in patients with advanced NSCLC and measure the improvement of survival and general symptoms in patients.

\section{Subjects and methods}

Patient eligibility. Between August 2007 and September 2010, 13 patients with histologically or cytologically confirmed locally advanced or metastatic NSCLC (disease stage IV) were considered as candidates in this pilot study. Patients who enrolled in this study were in accordance with the eligibility criteria listed in Table I: expected survival time $\geq 2$ months, Karnofsky performance status (KPS) score between 30 and $60 \%$, not pregnant or children and disease stage IV according to the UICC TNM classification. Previous therapy, including surgery, chemotherapy, radiation therapy or combined treatment, were not included in the eligibility criteria, but patients must not have received these therapies for at least 1 month prior to enrollment in this study and have abandoned treatment after their disease recurred to be involved in this study. There were also requirements concerning the function of certain organs; patients with severe arrest of bone marrow (grade 3-4) were excluded, adequate hepatic and renal function were required and patients with severe heart disease were excluded.

The research procedures were performed with the consent of the patients and ethics committee approval and were in accordance with the Helsinki Declaration of 1975, as revised in 1983 .

Treatment schedule. The MFs exposure generated by permanent magnets was employed. The system was designed by Shandong Chaoruishi Medical Science and Technology Company (Zibo, China) and is a static MFs device, which consists of two magnetic heads. In each magnetic head, a pair of fan-shaped NdFeB permanent magnets, which generate MFs, are located on a circular iron plate. The two magnetic heads rotate together around the patient. The bottom magnetic head is driven by an electro-motor, while the bonding force of the bottom magnetic head causes the top magnet to rotate synchronously. The MFs of the device are alternative pulses which are regulated from 0.38 to $0.42 \mathrm{~T}$. The rotation speed of the magnetic heads is regulated from 300 to $600 \mathrm{r} / \mathrm{min}$. The frequency of the MFs was $0-50 \mathrm{~Hz}$. The treatment bed was made of plexiglass located between the two magnetic heads.
Table I. Patient eligibility criteria.

Histologically or cytologically confirmed NCSLC

Disease stage IV, according to the UICC TNM classification

Karnofsky performance status score $30-60 \%$

Patients or their family members abandoned treatment after disease recurrence

Expected survival time $\geq 2$ months

Appropriate hepatic, heart and renal function

No severe arrest of bone marrow (grade 3-4)

Informed consent

No pregnant females or children

NSCLC, non-small cell lung cancer.

When treatment started, patients laid on the treatment bed of the ELF-MFs machine. The area of treatment included the primary tumor site, metastatic sites and metastatic lymph nodes. The treatment schedule was as follows: subjects were exposed to $420 \mathrm{r} / \mathrm{min}, 0.4-\mathrm{T}$ MFs, $2 \mathrm{~h}$ per day, 5 days per week for 6-10 weeks.

Toxicity and side-effects were evaluated according to WHO criteria. Before and following ELF-MFs treatment, all 13 patients underwent clinical tests, including physical examination, routine analysis of blood, ECG and liver function, biochemical and kidney function tests in order to evaluate toxicity and side-effects of ELF-MFs. During the treatment process, patients were observed to determine whether they had severe side-effects. If intolerable side-effects were reported, the treatment was stopped.

Changes in general symptoms, including pleural effusion, shortness of breath, cancer pain, appetite, physical capacity, constipation and insomnia, were recorded during ELF-MFs treatment and 2 weeks after the completion of therapy.

Follow-up. The follow-up methods included outpatient service and telephone interview. Following ELF-MFs treatment, all 13 patients were followed up every 3 months in the first year and every 6 months in the second and third year until the mortality of the patient. The last date of follow-up was September 20, 2010 .

Statistical analysis. Survival time was calculated from the end of treatment to mortality or last follow-up and was expressed as months. Overall survival, the 1- and 2-year survival rates and mortality or last follow-up results were evaluated by the Kaplan-Meier method. Overall survival was measured from the date of completion of ELF-MFs treatment to the date of mortality or last follow-up. Data analyses were conducted with SPSS 17.0 statistical software package (SPSS, Inc., Chicago, IL, USA). $\mathrm{P}<0.05$ was considered to indicate a statistically significant result.

\section{Results}

Patient characteristics. Of these 13 subjects, 5 were female and 8 were male and the median age was 65 years (range, 51-85). The characteristics of the patients are summarised in 
Table II. Characteristics of NSCLC patients.

\begin{tabular}{ll}
\hline Characteristics & Number $(\mathrm{n}=13)$ \\
\hline Age (years), median (range) & $65(51-85)$ \\
Gender, n (\%) & \\
Male & $8(61.5)$ \\
Female & $5(38.5)$ \\
Previous therapy, n (\%) & \\
Chemotherapy only & $5(38.5)$ \\
Surgery only & $1(7.7)$ \\
Combined treatment & $2(15.4)$ \\
None & $5(38.5)$ \\
Site of metastasis, n $(\%)$ & \\
Lung & $1(7.7)$ \\
Bone & $2(15.3)$ \\
Liver & $1(7.7)$ \\
Peritoneal cavity & $1(7.7)$ \\
Multi-site metastases & $3(23.1)$ \\
None & $5(38.5)$ \\
\hline
\end{tabular}

NSCLC, non-small cell lung cancer.

Table III. Symptom improvement of NSCLC patients.

\begin{tabular}{ll}
\hline Variable & Number $(\%)$ \\
\hline Pleural effusion decreasing & $2(15.4)$ \\
Remission of shortness of breath & $5(38.5)$ \\
Relief of cancer pain & $5(38.5)$ \\
Increased appetite & $6(46.2)$ \\
Improved physical strength & $9(69.2)$ \\
Better sleep quality & $1(7.7)$ \\
Regular bowel movement & $2(15.4)$ \\
Palliation of symptoms & $2(15.4)$ \\
\hline
\end{tabular}

${ }^{\text {aThese }} 2$ patients presented with palliation of symptoms during treatment, which disappeared at treatment termination. NSCLC, non-small cell lung cancer.

Table II. All patients suffered locally advanced or metastatic disease. Of these patients, 5 had single-site metastasis (lung, bone, liver and peritoneal cavity), 3 had multi-site metastases and 5 had no metastasis. Before the study, a total of 5 patients had not received anti-tumor treatment and 8 patients had received treatment, including chemotherapy, surgery or combined treatment. All the subjects experienced recurrence, and they abandoned treatment before receiving ELF-MFs.

Palliation of general symptoms. After the treatment, the clinical symptoms of all patients were investigated. The improvement of the general symptoms of all subjects are shown in Table III. Before ELF-MFs treatment, 2 patients had pleural effusion and a decrease in pleural effusion was observed in these cases at the end of treatment. A total of
Table IV. Toxicity and side-effects in NSCLC patients.

\begin{tabular}{ll}
\hline Variable & Number $(\%)$ \\
\hline Grade 1 arrest of bone marrow & $2(15.3)$ \\
Grade 2 arrest of bone marrow & $1(7.7)$ \\
Increased heart rate of $3-5 \mathrm{bpm}$ & $2(15.4)$ \\
Increased temperature of $0.5-1.0^{\circ} \mathrm{C}$ & $3(23.1)$
\end{tabular}

NSCLC, non-small cell lung cancer.

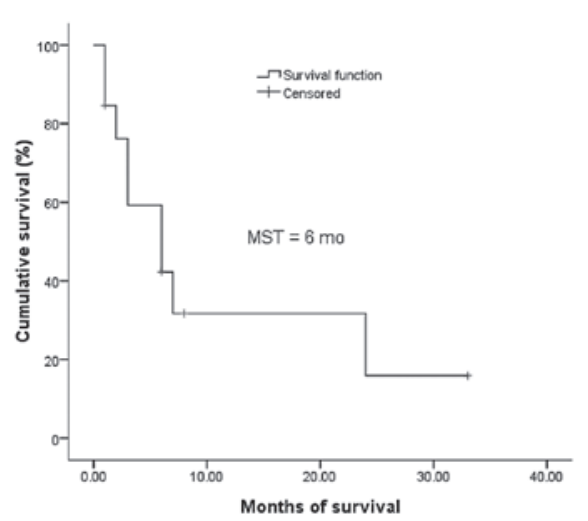

Figure 1. Cumulative survival curve of advanced NSCLC patients. NSCLC, non-small cell lung cancer; MST, median survival time.

5 patients reported remission of shortness of breath in the post-treatment period compared with pre-therapy of ELF-MFs. A total of 5 patients reported that magnetic treatment relieved cancer pain to various degrees. Lack of appetite is common in patients with advanced neoplasia; 6 patients in this study stated that they had increased appetite after treatment. One patient each reported constipation and irregular bowel movement before the treatment, and their bowel habit became regular, with once every 1-2 and 2-3 days, respectively, following treatment. Other clinical symptoms, including improved physical strength and better sleep quality, were detected in 9 and 1 patients, respectively. During the ELF-MFs treatment, 2 patients presented with improved symptoms during therapy, while the palliation of symptoms disappeared at treatment termination.

Toxicity and side-effects. Grades 1 and 2 arrest of bone marrow was detected in $2(15.4 \%)$ patients and $1(7.7 \%)$ patient, respectively. Increased heart rate of 3-5 bpm was observed in $2(15.4 \%)$ patients. There was an increase of temperature of $0.5-1.0^{\circ} \mathrm{C}$ in $3(23.1 \%)$ patients (Table IV). No severe sideeffects or toxicity were detected in the 13 advanced NSCLC patients treated by ELF-MFs in our trial.

Survival analysis. The 13 patients were all followed up either for 33 months or until mortality. The follow-up rate was $100.0 \%$. At the end of follow-up, 9 patients had succumbed to the disease, 1 patient was lost to follow-up and 3 patients were still alive. The median survival was 6.0 months $(95 \% \mathrm{CI}$, 1.0-11.0). The 1- and 2-year survival rates were 31.7 and $15.9 \%$, respectively (Fig. 1). 
Table V. Median survival and 1-year survival rate of ELF-MFssupportive care, cisplatin- and carboplatin-treated patients.

\begin{tabular}{lcc}
\hline Variable & $\begin{array}{c}\text { Median } \\
\text { survival (months) }\end{array}$ & $\begin{array}{c}\text { 1-year } \\
\text { survival rate (\%) }\end{array}$ \\
\hline ELF-MFs & 6.0 & 31.7 \\
Supportive care $^{\mathrm{a}}$ & 4.0 & 15.0 \\
Cisplatin-treated $^{\mathrm{b}}$ & 9.1 & 37.0 \\
Carboplatin-treated $^{\mathrm{b}}$ & 8.4 & 34.0 \\
\hline
\end{tabular}

${ }^{\mathrm{a}}$ Ardizzoni et al, reported in 2007 (19). ${ }^{\mathrm{b}}$ Reported in a meta-analysis in 1995 (18). ELF-MFs, extremely low-frequency magnetic fields.

\section{Discussion}

Lung cancer is one of the most common malignancies worldwide, both in incidence and mortality, and is the leading cause of cancer-related mortality in the world $(13,14)$. NSCLC accounts for approximately $80 \%$ of all lung tumors, $65-80 \%$ of which present as locally advanced or metastatic disease (15). For locally advanced or metastatic lung cancer, treatment is limited. Surgical excision is impossible in most patients, while supportive care and chemotherapy is widely used (14). Chemotherapy, either first- or second-line regimen, has low response rates and a high incidence of side-effects, which affect quality of life (QOL) outcomes of advanced or metastatic lung cancer patients $(16,17)$. MFs may be useful for the improvement of general symptoms and QOL outcomes of patients. It has been reported that MFs with specific physical characteristics may be safely used to treat patients with advanced neoplasia, and the patients have a good tolerability $(5,9)$.

We reviewed the English language literature and found that studies mainly focused on basic experimental studies, while only one clinical study was found to assess the safety of MFs treatment for patients with cancer (5). However, at present, no clinical trial to investigate the effects of ELF-MFs on survival and palliation of general symptoms in patients with advanced neoplasia has been performed. The aim of this study was to evaluate the improvement of general symptoms and survival in 13 advanced NSCLC patients treated by ELF-MFs.

Our results showed that the median survival was 6.0 months (95\% CI, 1.0-11.0). A meta-analysis (18) reported that the median survival of the advanced NSCLC patients receiving supportive care was 4.0 months. This study demonstrated that the median survival of patients treated with ELF-MFs was longer than that of those receiving supportive care. Our median survival of 6.0 months was less than that of 9.1 and 8.4 months of cisplatin- and carboplatin-treated advanced NSCLC patients, respectively (19). Furthermore, the 1-year survival rate was 31.7 in our ELF-MFs trial, which was lower than the 37 and 34\% reoprted in cisplatin- and carboplatintreated patients (19). However, the 1-year survival rate in ELF-MFs-treated advanced NSCLC patients was higher than that reported in the meta-analysis (18), whose 1-year survival rate in patients who received supportive care was $15 \%$. The median survival and 1-year survival rate of ELF-MFs-, supportive care, cisplatin- and carboplatin-treated patients are listed in Table V. According to the comparison of the median survival and the 1-year survival rate between ELF-MFs and supportive care treatments, we conclude that the advanced NSCLC patients may benefit from ELF-MFs treatment. Although the median survival and the 1-year survival rate in the ELF-MFs trial is no more than that of chemotherapy treatment (18-20), there are lower levels of toxicity and excellent tolerability in patients treated with ELF-MFs. In chemotherapy treatment for advanced NSCLC patients, toxicity includes neutropenia, thrombocytopenia, diarrhea, nausea, vomiting and fatigue $(16,17,21,22)$. However, in our trial, 2 (15.4\%) patients had grade 1 arrest of bone marrow and $1(7.7 \%)$ patient had grade 2. Increased heart rate of 3-5 bpm and temperature increase of $0.5-1.0^{\circ} \mathrm{C}$ was observed in $2(15.4 \%)$ and $3(23.1 \%)$ patients, respectively. We concluded that no severe toxicity or side-effects were detected in ELF-MFs treatment, which was in accordance with previous studies (5).

For advanced, especially stage IV, NSCLC patients in our trial, the chance of attaining complete remission was limited. It was essential to prolong survival (16) and improve general symptoms, which may lead to improvement of QOL in these patients. However, chemotherapy-induced side-effects remain a significant clinical problem in advanced NSCLC (16). It has been previously documented that cancer patients who undergo chemotherapy experience treatment-related symptoms and side-effects, including nausea, insomnia, diarrhea and diminished physical capacity $(16,17,21,22)$. By contrast, in the ELF-MFs treatment, the patients showed improvements in pleural effusion, shortness of breath, cancer pain, appetite, physical capacity, bowel movement and sleep quality in a certain number of patients. This finding demonstrates that ELF-MFs may moderately improve general symptoms in advanced NSCLC.

In conclusion, the results of our pilot trial with a small number of patients suggest that ELF-MFs may be an effective, well-tolerated and safe method of treatment of advanced NSCLC to prolong survival and moderately improve general symptoms. This is the first study to describe survival and palliation of general symptoms and the results merit further studies.

\section{Acknowledgements}

This study was supported by the program of 'Science and Technology Development Plan of Shandong Province of China' (No. 2011GSF11843).

\section{References}

1. Huang L, Dong L, Chen Y, Qi H and Xiao D: Effects of sinusoidal magnetic field observed on cell proliferation, ion concentration, and osmolarity in two human cancer cell lines. Electromagn Biol Med 25: 113-126, 2006.

2. Tofani S, Barone D, Berardelli M, Berno E, Cintorino M, Foglia L, Ossola P, Ronchetto F, Toso E and Eandi M: Static and ELF magnetic fields enhance the in vivo anti-tumor efficacy of cis-platin against lewis lung carcinoma, but not of cyclophosphamide against B16 melanotic melanoma. Pharmacol Res 48: 83-90, 2003.

3. Novikov VV, Novikov GV and Fesenko EE: Effect of weak combined static and extremely low-frequency alternating magnetic fields on tumor growth in mice inoculated with the Ehrlich ascites carcinoma. Bioelectromagnetics 30: 343-351, 2009. 
4. Zhang X, Zhang H, Zheng C, Li C, Zhang X and Xiong W: Extremely low frequency (ELF) pulsed-gradient magnetic fields inhibit malignant tumour growth at different biological levels. Cell Biol Int 26: 599-603, 2002.

5. Ronchetto F, Barone D, Cintorino M, Berardelli M, Lissolo S, Orlassino R, Ossola P and Tofani S: Extremely low frequency-modulated static magnetic fields to treat cancer: A pilot study on patients with advanced neoplasm to assess safety and acute toxicity. Bioelectromagnetics 25: 563-571, 2004.

6. Elson E: I. The little explored efficacy of magnetic fields in cancer treatment and postulation of the mechanism of action. Electromagn Biol Med 28: 275-282, 2009.

7. Ruiz-Gómez MJ and Martínez-Morillo M: Electromagnetic fields and the induction of DNA strand breaks. Electromagn Biol Med 28: 201-214, 2009

8. Lee HJ, Gimm YM, Choi HD, Kim N, Kim SH and Lee YS Chronic exposure of Sprague-Dawley rats to $20 \mathrm{kHz}$ triangular magnetic fields. Int J Radiat Biol 86: 384-389, 2010.

9. Wang T, Nie Y, Zhao S, Han Y, Du Y and Hou Y: Involvement of midkine expression in the inhibitory effects of low-frequency magnetic fields on cancer cells. Bioelectromagnetics 32: 443-452, 2011.

10. de Seze R, Tuffet S, Moreau JM and Veyret B: Effects of $100 \mathrm{mT}$ time varying magnetic fields on the growth of tumors in mice. Bioelectromagnetics 21: 107-111, 2000.

11. Tofani S, Barone D, Cintorino M, de Santi MM, Ferrara A, Orlassino R, Ossola P, Peroglio F, Rolfo K and Ronchetto F: Static and ELF magnetic fields induce tumor growth inhibition and apoptosis. Bioelectromagnetics 22: 419-428, 2001.

12. Hisamitsu T, Narita K, Kasahara T, Seto A, Yu Y and Asano K Induction of apoptosis in human leukemic cells by magnetic fields. Jpn J Physiol 47: 307-310, 1997.

13. Hu Z, Chen X, Zhao Y, Tian T, Jin G, Shu Y, Chen Y, Xu L, Zen K, Zhang C and Shen H: Serum microRNA signatures identified in a genome-wide serum microRNA expression profiling predict survival of non-small-cell lung cancer. J Clin Oncol 28: $1721-1726,2010$

14. Gao G, Chu H, Zhao L, Gui T, Xu Q and Shi J: A meta-analysis of paclitaxel-based chemotherapies administered once every week compared with once every 3 weeks first-line treatment of advanced non-small-cell lung cancer. Lung Cancer 76: 380-386, 2012.
15. Novello S and Le Chevalier T: Chemotherapy for non-small-cell lung cancer. Part 1: Early-stage disease. Oncology (Williston Park) 17: 357-364, 2003.

16. Guo L, Bai SP, Zhao L and Wang XH: Astragalus polysaccharide injection integrated with vinorelbine and cisplatin for patients with advanced non-small cell lung cancer: effects on quality of life and survival. Med Oncol: Sep 18, 2011 (E-pub ahead of print).

17. Adamsen L, Quist M, Midtgaard J, Andersen C, Møller T, Knutsen L, Tveterås A and Rorth $\mathrm{M}$ : The effect of a multidimensional exercise intervention on physical capacity, well-being and quality of life in cancer patients undergoing chemotherapy. Support Care Cancer 14: 116-127, 2006.

18. No authors listed: Chemotherapy in non-small cell lung cancer: a meta-analysis using updated data on individual patients from 52 randomised clinical trials. Non-small Cell Lung Cancer Collaborative Group. BMJ 311: 899-909, 1995.

19. Ardizzoni A, Boni L, Tiseo M, Fossella FV, Schiller JH, Paesmans M, Radosavljevic D, Paccagnella A, Zatloukal P, Mazzanti P, Bisset D and Rosell R; CISCA (CISplatin versus CArboplatin) Meta-analysis Group: Cisplatin- versus carboplatin-based chemotherapy in first-line treatment of advanced non-small-cell lung cancer: an individual patient data metaanalysis. J Natl Cancer Inst 99: 847-857, 2007.

20. Chiappori A, Bepler G, Barlesi F, Soria JC, Reck M, Bearz A, Barata F, Scagliotti G, Park K, Wagle A, Liepa AM, Zhao YD, Chouaki N, Iscoe $\mathrm{N}$ and von Pawel J: Phase II, double-blinded, randomized study of enzastaurin plus pemetrexed as second-line therapy in patients with advanced non-small cell lung cancer. J Thorac Oncol 5: 369-375, 2010.

21. Qi WX, Shen Z and Yao Y: Meta-analysis of docetaxel-based doublet versus docetaxel alone as second-line treatment for advanced non-small-cell lung cancer. Cancer Chemother Pharmacol 69: 99-106, 2012.

22. Qi WX, Tang LN, He AN, Shen Z and Yao Y: Effectiveness and safety of pemetrexed-based doublet versus pemetrexed alone as second-line treatment for advanced non-small-cell lung cancer: a systematic review and meta-analysis. J Cancer Res Clin Oncol 138: 745-751, 2012. 